Pontif́́cia U Uiversidade Católica $_{\text {a }}$

José Flávio Martins Cruz

\title{
CARACTERIZAÇÃO DE GASOLINAS POR ESPECTROSCOPIA FT- RAMAN
}

\author{
TESE DE DOUTORADO
}

Tese apresentada ao Programa de Pós-Graduação em Química do Departamento de Química da PUCRio como parte dos requisitos parciais para obtenção do título de Doutor em Química.

Orientadores: $\operatorname{Prof}^{\mathrm{a}} \mathrm{Dr}^{\mathrm{a}}$ Maria Isabel Pais da Silva e Prof. Dr. Claudio Alberto Téllez Soto 


\section{CARACTERIZAÇÃO DE GASOLINAS POR ESPECTROSCOPIA FT- RAMAN}

Tese apresentada como requisito parcial para obtenção do título de Doutor em Ciências-Química pelo Programa de Pós-Graduação em Química da PUC-Rio. Aprovada pela Comissão Examinadora abaixo assinada.

$$
\begin{array}{r}
\text { Profa . Dra . Maria Isabel Pais da Silva } \\
\text { Orientadora } \\
\text { Departamento de Química - PUC-Rio }
\end{array}
$$

Prof. Dr. Claudio Alberto Téllez Soto
Orientador
Departamento de Química - PUC-Rio

Prof. Dr. Dilson Norio Yshikawa

Universidade Estadual de Londrina

Prof. Dr. Ruy Luiz Milidin Departamento de Informática - PUC-Rio

Prof. Dr. Ricardo Queiroz Aurélio Departamento de Química - PUC-Rio

Prof. Dr. Carmen Lúcia Porto da Silveira Departamento de Química - PUC-Rio

Ney Augusto Dumont

Coordenador Setorial de Pós-Graduação e Pesquisa do Centro Técnico Científico - PUC-Rio

Rio de Janeiro, 31 de janeiro 2003 
Todos os direitos reservados. É proibida a reprodução total ou parcial do trabalho sem autorização da universidade, do autor e da orientadora.

\section{José Flávio Martins Cruz}

Graduou-se em Química na Faculdade de Filosofia Ciência e Letras da Universidade do Estado da Guanabara, em 1970. Especialista em Geoquímica pela Universidade Federal Fluminense, em 1993. Mestre em Química pela Universidade Católica do Rio de Janeiro, em 1998.

Ficha Catalográfica

Cruz, José Flávio Martins

Caracterização de gasolinas por espectroscopia FT-RAMAN / José Flávio Martins Cruz; orientadores: Maria Isabel Pais da Silva e Claudio Alberto Téllez Soto. - Rio de Janeiro : PUC, Departamento de Química, 2003.

[11], 215 f. : il. ; $30 \mathrm{~cm}$

Tese (doutorado) - Pontifícia Universidade Católica do Rio de Janeiro, Departamento de Química.

1. Química - Teses. 2. Espectroscopia Raman. 3. Petróleo. 4. Gasolina. 5. Mínimos quadrados parciais. I. Silva, Maria Isabel Pais da. II. Soto, Claudio Alberto Téllez. III. Pontifícia Universidade Católica do Rio de Janeiro. Departamento de Química. IV. Título. 


\section{Agradecimentos}

Agradeço a todos que conviveram comigo na dura, porém profícua e enriquecedora caminhada, que foi a produção deste trabalho, em especial:

À minha família, pela compreensão e incentivo irrestritos.

Ao Marcelo e Jorginho, meus irmãos, pelo apoio qualificado nas questões relativas à Estatísticas e à comunicação em Língua Portuguesa

À Inêz, minha noiva, pelo companheirismo e paciência em digitar, nas horas de lazer, a versão inicial desta tese.

À Elaine Luz, colega de pós-graduação, pela generosidade em me oferecer toda sua experiência sobre Mínimos Quadrados Parciais. Sem este apoio esta tese não teria se realizado.

Ao Prof. Dr. Claudio Alberto Téllez Soto, pelo entusiasmo, grande formação humana e pela crença profunda na necessidade de interação dos diversos matízes da produção do conhecimento humano, pelo domínio do tempo necessário para que uma aprendizagem se realize.

À Prof ${ }^{a} \operatorname{Dr}^{a}$ Maria Isabel Pais da Silva, pela compreensão e ajuda .

À PUC-RIO pela bolsa de isenção durante o período regular do programa de doutorado.

Aos professores e funcionários de apoio que, anonimamente, ajudam-nos na construção de nossos projetos.

À Fátima, secretária da pós-graduação, pelo eficaz apoio técnicoadministrativo.

Aos colegas de curso que, com suas alegrias e ansiedades mantêm vivo o clima de relações humanas que embora, às vezes, esquecido é meio de cultura fundamental para alcançarmos nossos objetivos. 


\section{Resumo}

Cruz, José Flávio Martins; Silva, Maria Isabel Pais da; Soto, Cláudio Alberto Téllez. Caracterização de gasolinas por espectroscopia FTRAMAN. Rio de Janeiro, 2003, 215p. Tese de Doutorado Departamento de Química, Pontifícia Universidade Católica do Rio de Janeiro.

Visando determinar os teores dos componentes relevantes e as propriedades físicas de gasolinas comerciais e sintéticas foram tomados espectros Raman de 60 gasolinas comerciais e 52 misturas sintéticas simulando gasolinas. Os espectros foram tomados em um espectrômetro Nicolet FT Raman 950. Os espectros brutos obtidos foram tratados para evitar a influência da variabilidade de potência do laser excitante sobre as intensidades das linhas Raman. As variáveis independentes (intensidades Raman ) e as variáveis dependentes (propriedades das gasolinas comerciais e misturas sintéticas ) foram centradas em torno da média e submetidas à regressão por mínimos quadrados parciais, visando ajustar modelos que permitissem predizer quantitativamente os teores de etanol, hidrocarbonetos saturados, insaturados e aromáticos além dos valores das propriedades MON, RON, densidade e pontos de ebulição inicial, final, a 10\%, 50\% e 90\% das amostras em estudo. Os resultados obtidos mostraram a potencialidade da espectroscopia Raman, para o desenvolvimento de métodos confiáveis para a análise de diversas características das gasolinas estudadas.

\section{Palavras-chave}

Espectroscopia Raman; petróleo; gasolina; mínimos quadrados parciais. 


\section{Abstract}

Cruz, José Flávio Martins; Silva, Maria Isabel Pais da; Soto, Cláudio Alberto Téllez. Caracterização de gasolinas por espectroscopia FTRAMAN. Rio de Janeiro, 2003, ...p. Tese de Doutorado - Departamento de Química, Pontifícia Universidade Católica do Rio de Janeiro.

The aim of this work was to determine the contents of the more important components and physical properties of commercial gasolines and synthetic mixtures with known composition, prepared in the laboratory. The Raman spectra of 60 gasolines and 52 mixtures were acquired with a Nicolet 950 Fourier Transform Raman (FT-Raman) spectrometer. The raw spectra were treated to avoid the laser potency variability on Raman lines intensities. The independent variables (Raman intensities) and the dependent variables (gasolines and mixtures properties) were mean centered and models were fit by partial least square regression seeking to predict the contents of ethanol, saturated, unsaturated and aromatic hydrocarbons. Also properties as MON, RON, density and boiling point values were determined by this procedure. The final results showed the potential of Raman spectroscopy for analysis of several properties of gasolines.

\section{Keywords}

Raman Spectroscopy; petroleum; gasoline; partial least squares. 
Memória de Marina. Breve, porém marcante existência. 


\section{Sumário}

1. Introdução 12

2. Petróleo e gasolina $\quad 15$

2.1. Petróleo 15

2.2. Gasolina automotiva $\quad 19$

2.2.1 - Obtenção de Gasolina 20

2.2.2 - Tipos de Gasolina 25

2.2.3 - Características e Requisitos de desempenho 26

2.2.4 - Especificações.Métodos de Análise e Seus Significados 26

3 - Espectroscopia Molecular $\quad 41$

3.1 - Aspectos Gerais $\quad 41$

3.2 - Espectroscopia Vibracional $\quad 47$

3.2.1 - Vibrações Moleculares 49

3.2.2 - O Modelo Do Oscilador Harmonico Clássico 50

3.2.3 - Classificação das Vibrações 51

3.2.4 - A Espectroscopia Raman 53

3.2.5 - Análises Qualitativa e Quantitativa por Espectroscopia Raman $\quad 56$

3.2.5.1 - Análise Qualitativa 56

3.2.5.2 - Análise Quantitativa $\quad 59$

4. Revisão bibliográfica 61

5. Quimiometria 64

5.1 - Análise Exploratória de Dados $\quad 65$

5.2 - Calibração Multivariada 66

5.2.1 - Regressão por Mínimos Quadrados Parciais (PLSR) 67

6. Aspectos Experimentais $\quad 81$

6.1 - A Espectroscopia Raman com Transformada de Fourier (FT-Raman) 82

6.2 - Preparo das Amostras de Gasolina Sintéticas e comerciais Analisadas 84

6.2.1 - Amostra de Gasolina Sintetica $\quad 84$

6.2.2 - Amostra de Gasolinas comerciais $\quad 84$

7. Resultados $\quad 86$

7.1 - Misturas Sintéticas $\quad 88$

7.2 - Gasolinas comerciais $\quad 106$

8. Discussão e Conclusão $\quad 127$

9. Referências Bibliográfica $\quad 129$

$\begin{array}{ll}\text { Apêndice A } & 131\end{array}$

Apêndice B $\quad 135$

$\begin{array}{ll}\text { Apêndice C } & 171\end{array}$ 


\section{Lista de figuras}

Fig. 2.2.1.1 - Principais processos de refinação de gasolinas 20

Fig. 2.2.1.2 - Distribuição de hidrocarbonetos em gasolinas 24

Fig. 2.2.4.1 - Curva de Destilação de gasolinas 31

Fig. 3.2.3.1 - Vibrações moleculares normais $\quad 52$

Fig. 3.2.4.1 Espalhamento elástico e inelástico de uma radiação 54

Figura 3.2.4.2 - Linhas Stokes e anti-Stokes do $\mathrm{CCl}_{4}$ líquido 55

Figura 3.2.4.3 - Espalhamento elástico e inelástico de um radiação 56

Fig. 3.2.5.1.1 - Espectros de gasolinas e componentes puros 58

Fig. 5.2.1.1.1 - RMSEP e números de componentes principais para o benzeno em gasolinas comerciais $\quad 80$

Fig. 6.1.1 - Aspecto de um interferograma 82

Fig. 7.1.1 - Teor\% de Etanol nas misturas sintéticas 94

Fig. 7.1.2 - Teor\% de n-Heptano nas misturas sintéticas 95

Fig. 7.1.3 - Teor\% de isooctano nas misturas sintéticas 96

Fig. 7.1.4 - Teor\% de ciclo-hexano $\quad 97$

Fig. 7.1.5-Teor\% de saturados 98

Fig. 7.1.6-Teor\% de ciclo-hexeno 99

Fig. 7.1.7 - Teor\% de benzeno 100

Fig. 7.1.8 - Teor\% de tolueno 101

Fig. 7.1.9 - Teor\% de p-xileno 102

Fig. 7.1.10-Teor\% de m-xileno 103

Fig. 7.1.11 - Teor\% de o-xileno 104

Fig. 7.1.12 - Teor\% de aromáticos 105

Fig. 7.2.1 - Teor\% de saturados $\quad 112$

Fig. 7.2.2 - Teor\% de benzeno 113

Fig. 7.2.3 - Teor\% de aromáticos 114

Fig. 7.2.4 - Teor\% de olefinas 115

Fig. 7.2.5 - Número de octanos (mon) 116

Fig. 7.2.6 - Número de octanos pesquisa (ron) 117

Fig. 7.2.7 - Índice antidetonante (iad) 118

Fig. 7.2.8 - Densidade relativa 119

Fig. 7.2.9 - Ponto de ebulição inicial 120

Fig. 7.2.10 - Ponto de ebulição- 10\% 121

Fig. 7.2.11 - Ponto de ebulição- 50\% 122

Fig. 7.2.12 - Ponto de ebulição- 90\% 123

Fig. 7.2.13 - Ponto de ebulição final $\quad 124$

Fig. 7.2.14 - Teor\% em volume de etanol 125

Fig. 7.2.15 - Teor\% em volume de Etanol( referência IROX) 126 


\section{Lista de tabelas}

Tab. 2.1 - Impurezas oleofílicas $\quad 17$

Tab 2.2 - Regulamentação técnica CNP no 04/79 27

Tab. 2.3 - Características antidetonantes de combustíveis 36

Tab. 2.4 - Condições de operação em motores CFR 38

Tab. 2.5 - Aditivos comerciais de gasolinas $\quad 40$

Tab. 3.1.1 - Regiões do espectro eletromagnético 44

Tab. 7.1 - RMSEP e coeficientes de correlação. Misturas Sintéticas 86

Tab. 7.2 - RMSEP e coeficientes de correlação. Gasolinas Comerciais 87

Tab. A1 - Composição de gasolinas sintéticas 131

Tab. A2 - Composição de gasolinas comerciais 132

Tab. B1 - Teor \% de etanol - calibração 135

Tab. B2 - Teor \% de etanol - validação 136

Tab. B3 - Teor \% de etanol - predição 137

Tab. B4 - Teor \% de etanol - resultado do teste de normalidade de resíduo 137

Tab. B5 - Teor \% de n-heptano - calibração 138

Tab. B6 - Teor \% de n-heptano - validação 139

Tab. B7 - Teor \% de n-heptano - predição 140

Tab. B8 - Teor \% de n-heptano - resultado do teste de normalidade de resíduo 140

Tab. B9 - Teor \% de isooctano - calibração 141

Tab. B10 - Teor \% de isooctano - validação 142

Tab. B11 - Teor \% de isooctano - predição 143

Tab. B12 - Teor \% de isooctano - resultado do teste de normalidade de resíduo 143

Tab. B13 - Teor \% de ciclo-hexano - calibração 144

Tab. B14 - Teor \% de ciclo-hexano - validação 145

Tab. B15 - Teor \% de ciclo-hexano - predição 146

Tab. B16 - Teor \% de ciclo-hexano - resultado do teste de normalidade de resíduo 146

Tab. B17 - Teor \% de saturados - calibração 147

Tab. B18 - Teor \% de saturados - validação 148

Tab. B19 - Teor \% de saturados - predição 149

Tab. B20 - Teor \% de saturados - resultado do teste de normalidade de resíduo 149

Tab. B21 - Teor \% de ciclo-hexeno - calibração 150

Tab. B22 - Teor \% de ciclo-hexeno - validação 151

Tab. B23 - Teor \% de ciclo-hexeno - predição 152

Tab. B24 - Teor \% de ciclo-hexeno - resultado do teste de normalidade de resíduo 152

Tab. B25 - Teor \% de benzeno - calibração 153

Tab. B26 - Teor \% de benzeno - validação 154

Tab. B27 - Teor \% de benzeno - predição 155

Tab. B28 - Teor \% de benzeno - resultado do teste de normalidade de resíduo 155

Tab. B29 - Teor \% de tulueno - calibração 156

Tab. B30 - Teor \% de tulueno - validação 157

Tab. B31 - Teor \% de tulueno - predição 158

Tab. B32 - Teor \% de tulueno - resultado do teste de normalidade de resíduo 158

Tab. B33 - Teor \% de p-xileno - calibração 159

Tab. B34 - Teor \% de p-xileno - validação 160

Tab. B35 - Teor \% de p-xileno - predição 161

Tab. B36 - Teor \% de p-xileno - resultado do teste de normalidade de resíduo 161

Tab. B37 - Teor \% de m-xileno - calibração 162

Tab. B38 - Teor \% de m-xileno - validação 163

Tab. B39 - Teor \% de m-xileno - predição 164

Tab. B40 - Teor \% de m-xileno - resultado do teste de normalidade de resíduo 164

Tab. B41 - Teor \% de o-xileno - calibração 165

Tab. B42 - Teor \% de o-xileno - validação 166

Tab. B43 - Teor \% de o-xileno - predição 167

Tab. B44 - Teor \% de o-xileno - resultado do teste de normalidade de resíduo 167

Tab. B45 - Teor \% de aromático - calibração 168

Tab. B46 - Teor \% de aromático - validação 169

Tab. B47 - Teor \% de aromático - predição 170

Tab. B48 - Teor \% de aromático - resultado do teste de normalidade de resíduo 170

Tab. C1 - Teor \% de saturados - calibração 171

Tab. C2 - Teor \% de saturados - validação 172

Tab. C3 - Teor \% de saturados - predição 173 
Tab. C4 - Teor \% de saturados - resultado do teste de normalidade de resíduo

Tab. C5 - Teor \% de benzeno - calibração

Tab. C6 - Teor \% de benzeno - validação

Tab. C7 - Teor \% de benzeno - predição

Tab. C8 - Teor \% de benzeno - resultado do teste de normalidade de resíduo 176

Tab. C9 - Teor \% de aromáticos - calibração 177

Tab. C10 - Teor \% de aromáticos - validação

Tab. C11 - Teor \% de aromáticos - predição

$\begin{array}{ll}\text { - Teor \% de aromáticos - predição } & 179\end{array}$

Tab. C12 - Teor \% de aromáticos - resultado do teste de normalidade de resíduo 179

Tab. C13 - Teor \% de olefinas - calibração

Tab. C14 - Teor \% de olefinas - validação

Tab. C15 - Teor \% de olefinas - predição

Tab. C16 - Teor \% de olefinas - resultado do teste de normalidade de resíduo 182

Tab. C17 - Número de octanos motor (MON) - calibração

Tab. C18 - Número de octanos motor (MON) - validação

Tab. C19 - Número de octanos motor (MON) - predição

Tab. C20 - Número de octanos motor (MON) - resultado do teste de nomalidade 185

Tab. C21 - Número de octanos pesquisa (RON) - calibração 186

Tab. C22 - Número de octanos pesquisa (RON) - validação 187

Tab. C23 - Número de octanos pesquisa (RON) - predição 188

Tab. C24 - Número de octanos pesquisa (RON) - resultado do teste de nomalidade 188

Tab. C25 - Índice antidetonante - calibração

Tab. C26 - Índice antidetonante - validação

Tab. C27 - Índice antidetonante - predição

Tab $\mathrm{C28}$ - Indice antidetonante - resultado do teste de normalidade de resíduo

Tab. C29 - densidade relativa - calibração

Tab. C30 - densidade relativa - validação

Tab. C31 - densidade relativa - predição

Tab. C32 - densidade relativa - resultado do teste de normalidade de resíduo

Tab. C33 - Ponto de ebulição inicial - calibração 195

Tab. C34 - Ponto de ebulição inicial - validação 196

Tab. C35 - Ponto de ebulição inicial - predição

Tab. C36 - Ponto de ebulição inicial - resultado do teste de normalidade de resíduo 197

Tab. C37 - Ponto de ebulição 10\% destilado - calibração 198

Tab. C38 - Ponto de ebulição 10\% destilado - validação 199

Tab. C39 - Ponto de ebulição 10\% destilado - predição 200

Tab. C40 - Ponto de ebulição $10 \%$ destilado - resultado do teste de nomalidade 200

Tab. C41 - Ponto de ebulição 50\% destilado - calibração 201

Tab. C42 - Ponto de ebulição 50\% destilado - validação 202

Tab. C43 - Ponto de ebulição 50\% destilado - predição 203

Tab. C44 - Ponto de ebulição 50\% destilado - resultado do teste de nomalidade 203

Tab. C45 - Ponto de ebulição 90\% destilado - calibração 204

Tab. C46 - Ponto de ebulição 90\% destilado - validação 205

Tab. C47 - Ponto de ebulição 90\% destilado - predição 206

Tab. C48 - Ponto de ebulição $90 \%$ destilado - resultado do teste de nomalidade 206

Tab. C49 - Ponto de ebulição final - calibração 207

Tab. C50 - Ponto de ebulição final - validação 208

Tab. C51 - Ponto de ebulição final - predição 209

Tab. C52 - Ponto de ebulição final - resultado do teste denomalidade de resíduo 209

Tab. C53 - Teor \% de etanol em volume - calibração 210

Tab. C54 - Teor \% de etanol em volume - validação 211

Tab. C55 - Teor \% de etanol em volume - predição 212

Tab. C56 - Teor \% de etanol em volume - resultado do teste denomalidade de resíduo 212

Tab. C57 - Teor \% de etanol IROX - calibração 213

Tab. C58 - Teor \% de etanol IROX - validação 214

Tab. C59 - Teor \% de etanol IROX - predição 215

Tab. C60 - Teor \% de etanol IROX - resultado do teste de normalidade de resíduo 215 